\title{
Vibration of Three-Layered FGM Cylindrical Shells with Middle Layer of Isotropic Material for Various Boundary Conditions
}

\author{
Muhammad Nawaz Naeem1, Awais Gul Khan1, Shahid Hussain Arshad2*, \\ Abdul Ghafar Shah ${ }^{3}$, Madiha Gamkhar ${ }^{4}$ \\ ${ }^{1}$ Department of Mathematics, G. C. University Faisalabad, Faisalabad, Pakistan \\ ${ }^{2}$ Aerospace Research Institute and North-West Composites Centre, University of Manchester, Manchester, UK \\ ${ }^{3}$ Department of Mathematics, Government Post Graduate College Jhang, Jhang, Pakistan \\ ${ }^{4}$ Department of Statistics and Mathematics, Agricultural University Faisalabad, Faisalabad, Pakistan \\ Email: shahid26188@yahoo.com
}

Received 26 September 2014; revised 21 October 2014; accepted 8 November 2014

Copyright (C) 2014 by authors and Scientific Research Publishing Inc.

This work is licensed under the Creative Commons Attribution International License (CC BY).

http://creativecommons.org/licenses/by/4.0/

c) (i) Open Access

\begin{abstract}
In the present study, vibration analysis of a three-layered cylindrical shell is performed whose inner and outer layers are composed of functionally graded materials whereas the middle one is assumed to be of isotropic material. This formation of a cylindrical shell influences stiffness modulii and the resultant material properties. The shell problem is formulated from the constitutive relations of stresses and strains with the displacement deformations and they are taken from Love's thin shell theory. This problem is transformed into the integral form by evaluating the expressions for the strain and kinetic energies of the shell. Rayleigh-Ritz method is employed to solve the shell dynamic equations. Vibration characteristics of these cylindrical shells are investigated for a number of physical parameters and configurations of the fabrication of shells. The axial modal dependence is approximated by the characteristic beam functions that satisfy the boundary conditions. Results evaluated, show good agreement with the open literature.
\end{abstract}

\section{Keywords}

Functionally Graded Material, Isotropic Material, Three-Layered Cylindrical Shell, Love's Thin Shell Theory Rayleigh-Ritz Method, Natural Frequency

\footnotetext{
${ }^{*}$ Corresponding author.
}

How to cite this paper: Naeem, M.N., et al. (2014) Vibration of Three-Layered FGM Cylindrical Shells with Middle Layer of Isotropic Material for Various Boundary Conditions. World Journal of Mechanics, 4, 315-331. 


\section{Introduction}

Yamanouchi et al. [1] introduced the concept of functionally graded materials (FGMs), working as aerospace researchers in Japan. Rabin and Heaps [2] reported some methods for the manufacturing of FGMs. The idea of ceramic transactions for the fabrication of FGMs was introduced by Koizumi [3]. Miyamoto et al. [4] composed a book on FGMs, in which they gave a high quality discussion on the design and applications of FGMs. Rayleigh [5] analyzed the study of Sophie on the vibration of circular cylindrical shells. Love [6] at the end of $19^{\text {th }}$ century, gave the first linear shell theory based on Krichhoff's hypothesis for plates. Arnold and Warburton [7] [8] derived equations of motion for vibration of thin circular cylindrical shells. They used Lagrange equations with strain and kinetic energy expressions to derive these equations. Forsberg [9] studied shell equations to scrutinize the effect of boundary conditions on vibration characteristics of circular cylindrical shells. In his work, exponential axial modal dependence was measured. Sewall and Naumann [10] studied analytical and experimental frequencies and mode shapes for the vibrations using the Rayleigh-Ritz method. The characteristic beam functions were used to approximate the modal dependence in the axial direction. Warburton [11], Warburton and Higgs [12] and Goldman [13] studied the natural frequencies and mode shapes of thin cylindrical shells and selected exponential functions for the modal dependence in the axial direction. Sharma [14] explored the natural frequencies of fixed free circular cylindrical shells. He [15] also studied the problems of vibration characteristics of thin circular cylindrical shells with various end conditions with first order shell theory of Sanders. A simple variational technique was applied to give a cubic frequency equation. Loy and Lam [16] studied the vibration of thin cylindrical shells with ring supports, placed along the shell length and which imposed a zero lateral deflection. The study was carried out using Sander's shell theory. Naeem and Sharma [17] employed an analytical procedure to study the free vibration characteristics of thin cylindrical shells. Ritz polynomial functions were assumed to satisfy the axial modal dependence and the Rayleigh Ritz variational approach was employed to formulate the general eigenvalue problem. Influence of some commonly used boundary conditions and shell parameters on the vibration frequencies were examined. Loy et al. [18] studied the vibration of FGM cylindrical shell fabricated with the constituent materials stainless steel and nickel. They concluded that the frequency characteristics were similar to those of homogeneous isotropic cylindrical shells and the frequencies were affected by the constituent volume fractions and the configurations of the constituent materials but they found the response of frequencies of FGM cylindrical shells for only simply supported boundary condition. This work was extended by Pradhan et al. [19] by studying the vibration of FGM cylindrical shell for various boundary conditions. Arshad et al. [20] [21] calculated natural frequencies of the FGM cylindrical shell by various volume fraction laws and under various boundary conditions respectively. Najafizadeh and Isvandzibaei [22] studied the vibration of thin FGM cylindrical shells with ring supports. The study was carried out based on third order shear deformation shell theory. The objective was to observe the influence of the configurations of the constituent materials, positions of the ring support and different boundary conditions on the natural frequencies of the cylindrical shells. The analysis was carried out with strain displacement relations from Love's shell theory. The governing equations were obtained using energy functional with the Rayleigh Ritz method. Sofiyev et al. [23] studied the vibration and stability analysis of a three-layered conical shell with middle layer composed of functionally graded material. They applied Galerkin numerical technique to transform the governing equations of motion into a pair of time dependent partial differential equations. They concluded that the material parameters are directly affected by the diverse configurations of the FGM constituents and by the nature of materials used in the shell layers. He [24] extended this work to study the vibration and stability response of a composite cylindrical shell containing a functionally graded layer. Li and Batra [25] [26] investigated other dynamic aspects of shells like buckling of a three layered simply supported axially compressed laminated composite as well as isotropic thin cylindrical shells. They designed these shells in such a way that the inner and outer layers of the shells were composed of the composite and isotropic materials respectively and a layer of functionally graded material was inserted at the middle in the transverse direction. Arshad et al. [27] [28] studied the frequency spectra of bi-layered cylindrical shells by taking different materials in both layers such as isotropic as well as functionally graded materials and by taking two different functionally graded materials at the inner and outer layers of the cylindrical shells respectively.

\section{Formulation of Shell Problem}

\subsection{Volume Fraction Law}

Most of functionally graded materials are used in high temperature and possess temperature dependent proper- 
ties. The material property $P$ is expressed as a function temperature $T(K)$ by Touloukian [29] as:

$$
P=P_{0}\left(P_{-1} T^{-1}+1+P_{1} T+P_{2} T^{2}+P_{3} T^{3}\right)
$$

where $P_{0}, P_{1}, P_{2}$ and $P_{3}$ are the coefficients of temperature $T(K)$ expressed in Kelvin and are unique to the constituent materials. The material properties $P$ of FGMs are a function of the material properties and volume fractions of the constituents, and can be expressed as:

$$
P=\sum_{j=1}^{k} P_{j} V_{f j}
$$

where $P_{j}$ and $V_{f j}$ are the material property and volume fraction of the constituent material $j$ respectively. For three-layered FGM cylindrical shells, the outer and inner layers are assumed to be functionally graded and the middle layer isotropic. The shell thickness is assumed to be distributed in three portions. With this assumption, the extensional, coupling and bending stiffness are modified in three layers. Let $M_{\text {in }}$ and $M_{\text {out }}$ represent the inner and outer constituent materials of the FGM layers used to fabricate the three layered FGM cylindrical shell with middle layer of isotropic material. For a functionally graded material layers, consisting of two materials $M_{\text {in }}$ and $M_{\text {out }}$, volume fraction is written for an effective material property as:

$$
P(z)=\left(P_{\text {in }}-P_{\text {out }}\right) V_{\text {in }}+P_{\text {out }}
$$

where $V_{\text {in }}$, the volume fraction, is defined for a material $M_{\text {in }}$ as:

$$
V_{\text {in }}=\left(\frac{z-h_{\text {in }}}{h_{\text {out }}-h_{\text {in }}}\right)^{p}
$$

where $z$ is the radial variable, $h_{\text {in }}$ and $h_{\text {out }}$ are the inner and outer coordinates from the centre of the circular cylindrical shell. Also $P_{\text {in }}$ and $P_{\text {out }}$ are the material properties of $M_{\text {in }}$ and $M_{\text {out }}$ respectively.

\subsection{Theoretical Considerations}

Consider a cylindrical shell as shown in the Figure 1(a). Let $R$ is the radius, $L$ is the length and $h$ is the thickness of the cylindrical shell. The orthogonal coordinates system $(x, \theta, z)$ is taken to be at the middle surface of the shell. The $x$-coordinate is taken in the axial direction of the shell $\theta$ is in the circumferential and $z$-coordinate is in the radial direction of the shell. The deformations of the shell in axial, circumferential and radial directions are represented by $u(x, \theta, t), v(x, \theta, t)$ and $w(x, \theta, t)$ respectively. For the study of thin cylindrical shell, three dimensional problems are converted in to two dimensional by applying plane stress condition. The constitutive relation of stress and strain of a thin cylindrical shell is given by Hook's law as:

$$
\{\sigma\}=[Q]\{e\}
$$

where $\{\sigma\}$ is the stress vector, $\{e\}$ is the strain vector and $[Q]$ is the reduced stiffness matrix. The stress vector and the strain vectors are defined as:

$$
\begin{aligned}
& \{\sigma\}^{\mathrm{T}}=\left\{\sigma_{x}, \sigma_{\theta}, \sigma_{x \theta}\right\} \\
& \{e\}^{\mathrm{T}}=\left\{e_{x}, e_{\theta}, e_{x \theta}\right\}
\end{aligned}
$$

where $\sigma_{x}$ and $\sigma_{\theta}$ are the normal stresses in $x$ and $\theta$ directions, and $\sigma_{x \theta}$ is the shear stress on the $x \theta$ -plane. Similarly $e_{x}$ and $e_{\theta}$ are the normal strains in the $x$ and $\theta$ directions, and $e_{x \theta}$ is the shear strain on the $x \theta$-plane. The reduced stiffness matrix is defined as:

$$
[Q]=\left[\begin{array}{ccc}
Q_{11} & Q_{12} & 0 \\
Q_{12} & Q_{22} & 0 \\
0 & 0 & Q_{66}
\end{array}\right]
$$




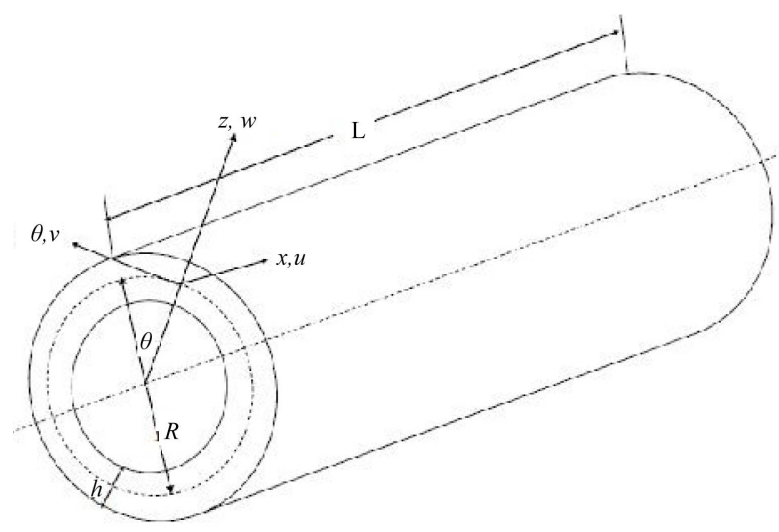

(a)

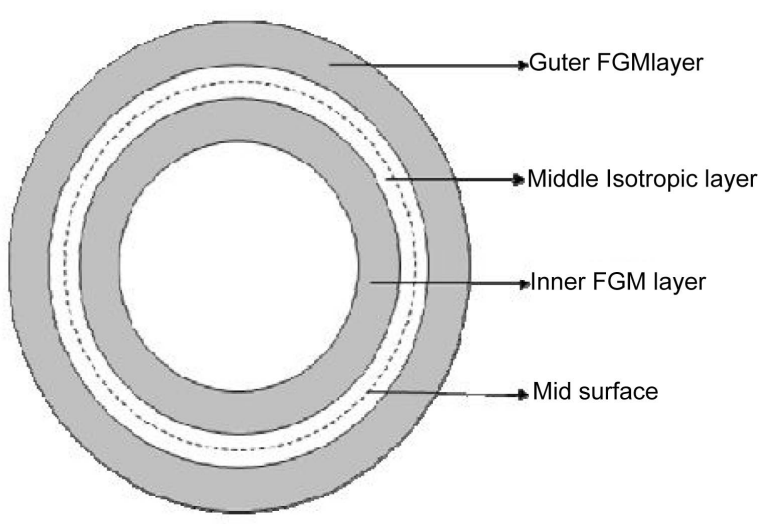

(b)

Figure 1. (a) Geometry of a Circular Cylindrical Shell; (b) Cross-sectional vision of three-layered cylindrical shell.

So the relation (5) can be expressed as:

$$
\left\{\begin{array}{c}
\sigma_{x} \\
\sigma_{\theta} \\
\sigma_{x \theta}
\end{array}\right\}=\left[\begin{array}{ccc}
Q_{11} & Q_{12} & 0 \\
Q_{12} & Q_{22} & 0 \\
0 & 0 & Q_{66}
\end{array}\right]\left\{\begin{array}{c}
e_{x} \\
e_{\theta} \\
e_{x \theta}
\end{array}\right\}
$$

For isotropic materials the reduced stiffness $Q_{i j}$ are defined as:

$$
Q_{11}=Q_{22}=\frac{E}{1-v^{2}}, \quad Q_{12}=\frac{v E}{1-v^{2}}, \quad Q_{66}=\frac{E}{2(1+v)}
$$

where $E$ is the Young's modulus and $v$ is the Poisson's ratio. According to Love's shell theory, the components in the strain vector $\{e\}$ are defined as:

$$
\left.\begin{array}{l}
e_{x}=e_{1}+z \kappa_{1} \\
e_{\theta}=e_{2}+z \kappa_{2} \\
e_{x \theta}=\gamma+2 z \tau
\end{array}\right\}
$$

where $e_{1}, e_{2}$ and $\gamma$ are the reference surface strains. $\kappa_{1}, \kappa_{2}$ and $\tau$ are the surface curvatures. From Equations (9) and (11) the components in the stress vector $\{\sigma\}$ are defined as:

$$
\left.\begin{array}{l}
\sigma_{x}=\left(e_{1}+z \kappa_{1}\right) Q_{11}+\left(e_{2}+z \kappa_{2}\right) Q_{12} \\
\sigma_{\theta}=\left(e_{1}+z \kappa_{1}\right) Q_{12}+\left(e_{2}+z \kappa_{2}\right) Q_{22} \\
\sigma_{x \theta}=(\gamma+2 z \tau) Q_{66}
\end{array}\right\}
$$

For a thin cylindrical shell the force and moment resultants are defined as:

$$
\begin{aligned}
& \left\{N_{x}, N_{\theta}, N_{x \theta}\right\}=\int_{\frac{-h}{2}}^{\frac{h}{2}}\left\{\sigma_{x}, \sigma_{\theta}, \sigma_{x \theta}\right\} \mathrm{d} z \\
& \left\{M_{x}, M_{\theta}, M_{x \theta}\right\}=\int_{\frac{-h}{2}}^{\frac{h}{2}}\left\{\sigma_{x}, \sigma_{\theta}, \sigma_{x \theta}\right\} z \mathrm{dz} z
\end{aligned}
$$

where $N_{x}, N_{\theta}$ and $N_{x \theta}$ are force components in axial, circumferential and shear directions. $M_{x}, M_{\theta}$ and $M_{x \theta}$ are moment components in axial, circumferential and shear directions. Equations (12), (13) and (14) implies: 


$$
\{N\}=[S]\{\in\}
$$

where $\{N\}$ and $\{\in\}$ are defined as:

$$
\begin{gathered}
\{N\}^{\mathrm{T}}=\left\{N_{x}, N_{\theta}, N_{x \theta}, M_{x}, M_{\theta}, M_{x \theta}\right\} \\
\{\in\}^{\mathrm{T}}=\left\{e_{1}, e_{2}, \gamma, \kappa_{1}, \kappa_{2}, 2 \tau\right\}
\end{gathered}
$$

and $[S]$ is defined as:

$$
[S]=\left[\begin{array}{ll}
{[A]} & {[B]} \\
{[B]} & {[D]}
\end{array}\right]
$$

where $[A],[B]$ and $[D]$ are the extensional, coupling and bending stiffness matrices given as:

$$
[A]=\left[\begin{array}{ccc}
A_{11} & A_{12} & 0 \\
A_{12} & A_{22} & 0 \\
0 & 0 & A_{66}
\end{array}\right] \quad[B]=\left[\begin{array}{ccc}
B_{11} & B_{12} & 0 \\
B_{12} & B_{22} & 0 \\
0 & 0 & B_{66}
\end{array}\right] \quad[D]=\left[\begin{array}{ccc}
D_{11} & D_{12} & 0 \\
D_{12} & D_{22} & 0 \\
0 & 0 & D_{66}
\end{array}\right]
$$

where $A_{i j}, B_{i j}$ and $D_{i j}$ are the extensional, coupling and bending stiffness and defined as:

$$
\left(A_{i j}, B_{i j}, D_{i j}\right)=\int_{\frac{-h}{2}}^{\frac{h}{2}}\left(1, Z, Z^{2}\right) Q_{i j} \mathrm{~d} z
$$

The coupling stiffness $B_{i j}$ become zero for isotropic cylindrical shell and is non-zero for FGM cylindrical shells. The general equations for strain energy and kinetic energy of a cylindrical shell can be written as:

$$
\begin{gathered}
U=\frac{1}{2} \int_{0}^{L} \int_{0}^{2 \pi}\{\in\}^{T}[S]\{\in\} R \mathrm{~d} \theta \mathrm{d} x \\
T=\frac{1}{2} \int_{0}^{L} \int_{0}^{L} \rho_{T}\left\{\left(\frac{\partial u}{\partial t}\right)^{2}+\left(\frac{\partial v}{\partial t}\right)^{2}+\left(\frac{\partial w}{\partial t}\right)^{2}\right\} R \mathrm{~d} \theta \mathrm{d} x
\end{gathered}
$$

where $\rho_{T}$ is the mass density per unit length and is defined as follows:

$$
\rho_{T}=\int_{\frac{-h}{2}}^{\frac{h}{2}} \rho \mathrm{d} z
$$

where $\rho$ is the mass density of the shell material.

By substituting $\{\in\},\{\in\}^{\mathrm{T}}$ and $[S]$ from Equations (17) and (18) in Equation (20) implies:

$$
\begin{gathered}
U=\frac{1}{2} \int_{0}^{L} \int_{0}^{2 \pi}\left\{A_{11} e_{1}^{2}+A_{22} e_{2}^{2}+2 A_{12} e_{1} e_{2}+A_{66} \gamma^{2}+2 B_{11} e_{1} \kappa_{1}+2 B_{12} e_{1} \kappa_{2}+2 B_{12} e_{2} \kappa_{1}+2 B_{22} e_{2} \kappa_{2}+4 B_{66} \gamma \tau+D_{11} \kappa_{1}^{2}\right. \\
\left.+D_{22} \kappa_{2}^{2}+2 D_{12} \kappa_{1} \kappa_{2}+4 D_{66} \tau^{2}\right\} R \mathrm{~d} \theta \mathrm{d} x .
\end{gathered}
$$

\subsection{Strain-Displacement and Curvature-Displacement Relation}

A number of shell theories have arisen and are used. Among these theories however the Love's shell theory is considered to be the first theory about shells and all other shell theories were derived from the Love's shell theory by amending some physical terms. The strain-displacement and the curvature-displacement relations which are adopted from Love's [6] shell theory are given as below:

$$
e_{1}=\frac{\partial u}{\partial x}, \quad e_{2}=\frac{1}{R}\left(\frac{\partial v}{\partial \theta}+w\right), \quad \gamma=\frac{\partial v}{\partial x}+\frac{1}{R} \frac{\partial u}{\partial \theta}
$$




$$
\kappa_{1}=-\frac{\partial^{2} w}{\partial x^{2}}, \quad \kappa_{2}=-\frac{1}{R^{2}}\left(\frac{\partial^{2} w}{\partial \theta^{2}}-\frac{\partial v}{\partial \theta}\right), \quad \tau=-\frac{1}{R}\left(\frac{\partial^{2} w}{\partial x \partial \theta}-\frac{\partial v}{\partial x}\right)
$$

By substituting these values of strain displacement and curvature displacement from Equations (24) and (25) in Equation (23), we obtain the strain energy equation in the form of displacement functions $u, v, w$ and their partial derivatives as:

$$
\begin{aligned}
U=\frac{1}{2} \int_{0}^{L} \int_{0}^{2 \pi}\left\{A_{11}(\right. & \left.\frac{\partial u}{\partial x}\right)^{2}+A_{22} \frac{1}{R^{2}}\left(\frac{\partial v}{\partial \theta}+w\right)^{2}+2 A_{12} \frac{1}{R}\left(\frac{\partial u}{\partial x}\right)\left(\frac{\partial v}{\partial \theta}+w\right)+A_{66}\left(\frac{\partial v}{\partial x}+\frac{1}{R} \frac{\partial u}{\partial \theta}\right)^{2}-2 B_{11}\left(\frac{\partial u}{\partial x}\right)\left(\frac{\partial^{2} w}{\partial x^{2}}\right) \\
& -2 B_{12} \frac{1}{R^{2}}\left(\frac{\partial u}{\partial x}\right)\left(\frac{\partial^{2} w}{\partial \theta^{2}}-\frac{\partial v}{\partial \theta}\right)-2 B_{12} \frac{1}{R}\left(\frac{\partial v}{\partial \theta}+w\right)\left(\frac{\partial^{2} w}{\partial x^{2}}\right)-2 B_{22} \frac{1}{R^{3}}\left(\frac{\partial v}{\partial \theta}+w\right)\left(\frac{\partial^{2} w}{\partial \theta^{2}}-\frac{\partial v}{\partial \theta}\right) \\
& -8 B_{66} \frac{1}{R}\left(\frac{\partial v}{\partial x}+\frac{1}{R} \frac{\partial u}{\partial \theta}\right)\left(\frac{\partial^{2} w}{\partial x \partial \theta}-\frac{\partial v}{\partial x}\right)+D_{11}\left(\frac{\partial^{2} w}{\partial x^{2}}\right)^{2}+D_{22} \frac{1}{R^{4}}\left(\frac{\partial^{2} w}{\partial \theta^{2}}-\frac{\partial v}{\partial \theta}\right)^{2} \\
+ & \left.2 D_{12} \frac{1}{R^{2}}\left(\frac{\partial^{2} w}{\partial x^{2}}\right)\left(\frac{\partial^{2} w}{\partial \theta^{2}}-\frac{\partial v}{\partial \theta}\right)+4 D_{66} \frac{1}{R^{2}}\left(\frac{\partial^{2} w}{\partial x \partial \theta}-\frac{\partial v}{\partial x}\right)^{2}\right\} R \mathrm{~d} \theta \mathrm{d} x
\end{aligned}
$$

\subsection{Lagrangian Energy Functional}

The Lagrangian energy functional $\Pi$, is the difference of the two types of shell energies and defined as:

$$
\Pi=T-U
$$

\subsection{Rayleigh-Ritz Method}

The energy variation methods i.e., Rayleigh Ritz and Galerkin methods are the most frequently used ones to analyze the shell vibrational behavior. In the Rayleigh Ritz method, the energy variational functional is minimized with respect to the coefficients of an approximating series representing the displacement deformations. Many researchers such as Sewall and Naumann [10], Sharma and Johns [15], Loy et al. [18] and Naeem and Sharma [17] used this procedure to analyze the vibration characteristics of the cylindrical shells.

\subsection{Axial Modal Dependence}

The expressions for the modal displacement deformations are presumed in the form of product of functions of space and time variables. This leads to a system of ordinary differential equations of three unknown functions of the axial space variable. Different types of functions are chosen to approximate the axial modal dependence. These functions satisfy the boundary conditions. Well-known functions are beam functions, Ritz polynomial functions, orthogonal polynomials and Fourier series of the circular functions. The expression for modal displacement deformations are assumed as:

$$
\begin{gathered}
u(x, \theta, t)=A \frac{\mathrm{d} \phi}{\mathrm{d} x} \cos n \theta \sin \omega t \\
v(x, \theta, t)=B \phi \sin n \theta \sin \omega t \\
w(x, \theta, t)=C \phi \cos n \theta \sin \omega t
\end{gathered}
$$

In the axial, circumferential and radial directions respectively, the coefficients $A, B$ and $C$ are the constants denoting the amplitudes of the vibrations in the $x, \theta$ and $z$ directions respectively, $n$ is the circumferential wave number and $\omega$ is the natural angular frequency for the cylindrical shell. The axial function $\phi(x)$ is chosen as the beam function as:

$$
\phi(x)=\alpha_{1} \cosh \left(\frac{\lambda_{m} x}{L}\right)+\alpha_{2} \cos \left(\frac{\lambda_{m} x}{L}\right)-\varsigma_{m}\left(\alpha_{3} \sinh \left(\frac{\lambda_{m} x}{L}\right)+\alpha_{4} \sin \left(\frac{\lambda_{m} x}{L}\right)\right)
$$


where $\alpha_{i}(i=1,4)$ are some constants with value 0 or 1 chosen according to the boundary condition. $\lambda_{m}$ are the roots of some transcendental equations and $\varsigma_{m}$ are some parameters dependent on $\lambda_{m}$. Their values are given as in Table 1 :

The geometric boundary conditions for clamped, free and simply supported boundary conditions can be expressed mathematically in terms of characteristic beam function $\phi(x)$ as:

Clamped boundary condition $\phi(x)=\phi^{\prime}(x)=0$

Free boundary condition $\phi^{\prime \prime}(x)=\phi^{\prime \prime \prime}(x)=0$

Simply supported boundary condition $\phi(x)=\phi^{\prime \prime}(x)=0$

\subsection{Derivation of Frequency Equation}

On substituting the expressions for the deformation displacements $u, v$ and $w$ in the expression for the strain and kinetic energies of the cylindrical shells and employing the principle of minimization of the energy the expression for maximum strain and kinetic energies are obtained. The new form of the Lagrangian functional is formed as:

$$
\Pi=T_{\max }-U_{\max }
$$

where

$$
\begin{gathered}
U_{\max }=\frac{\pi R}{2} \int_{0}^{L}\left[A_{11} A^{2}\left(\frac{\mathrm{d}^{2} \varphi}{\mathrm{d} x^{2}}\right)^{2}+\frac{1}{R^{2}} A_{22}(n B+C)^{2} \varphi^{2}+\frac{2}{R} A_{12}(n A B+A C) \varphi \frac{\mathrm{d}^{2} \varphi}{\mathrm{d} x^{2}}+A_{66}\left(B-\frac{n A}{R}\right)^{2}\left(\frac{\mathrm{d} \varphi}{\mathrm{d} x}\right)^{2}\right. \\
-2 B_{11} A C\left(\frac{\mathrm{d}^{2} \varphi}{\mathrm{d} x^{2}}\right)^{2}+\frac{2}{R^{3}} B_{22}(n B+C)\left(n^{2} C+n B\right) \varphi^{2}+\frac{2}{R^{2}} B_{12}\left(n^{2} A C+n A B\right) \varphi \frac{\mathrm{d}^{2} \varphi}{\mathrm{d} x^{2}} \\
-\frac{2}{R} B_{12}\left(n B C+C^{2}\right) \varphi \frac{\mathrm{d}^{2} \varphi}{\mathrm{d} x^{2}}+\frac{4}{R} B_{66}\left(B-\frac{n A}{R}\right)(n C+B)\left(\frac{\mathrm{d} \varphi}{\mathrm{d} x}\right)^{2}+D_{11} C^{2}\left(\frac{\mathrm{d}^{2} \varphi}{\mathrm{d} x^{2}}\right)^{2} \\
\left.+\frac{1}{R^{4}} D_{22}\left(n^{2} C+n B\right)^{2} \varphi^{2}-\frac{2}{R^{2}} D_{12}\left(n^{2} C^{2}+n B C\right) \varphi \frac{\mathrm{d}^{2} \varphi}{\mathrm{d} x^{2}}+\frac{4}{R^{2}} D_{66}(n C+B)^{2}\left(\frac{\mathrm{d} \varphi}{\mathrm{d} x}\right)^{2}\right] \mathrm{d} x . \\
T_{\max }=\frac{\pi R}{2} \rho_{T} \omega^{2} \int_{0}^{L}\left[\left(\frac{\mathrm{d} \phi}{\mathrm{d} x}\right)^{2} A^{2}+\phi^{2} B^{2}+\phi^{2} C^{2}\right] \mathrm{d} x
\end{gathered}
$$

To derive the shell frequency equation, the energy functional is extremized with respect to the vibration amplitudes: $A, B$ and $C$, resulting in three homogenous linear following equations:

$$
\frac{\partial \Pi}{\partial A}=\frac{\partial \Pi}{\partial B}=\frac{\partial \Pi}{\partial C}=0
$$

By re-arranging Equation (35), the shell frequency equation is written in the eigenvalue form as:

$$
\left[\begin{array}{lll}
C_{11} & C_{12} & C_{13} \\
C_{12} & C_{22} & C_{23} \\
C_{13} & C_{23} & C_{33}
\end{array}\right]\left[\begin{array}{l}
A \\
B \\
C
\end{array}\right]=\rho h \omega^{2}\left[\begin{array}{ccc}
I_{2} & 0 & 0 \\
0 & I_{4} & 0 \\
0 & 0 & I_{4}
\end{array}\right]\left[\begin{array}{l}
A \\
B \\
C
\end{array}\right]
$$

where the expressions for the terms $C_{i j}$ 's, $I_{2}$ and $I_{4}$ are the given in Appendix.

\section{Results and Discussion}

A number of comparison of the results for isotropic and FGM cylindrical shells are presented to verify the validity, efficiency and accuracy of the present approach. The present analysis is carried out by using the energy variational procedure viz: Rayleigh-Ritz method. This method is based on the principle of minimization of energy. The numerical results for the following three frequently encountered sets of boundary conditions are evaluated to check the validity, efficiency and accuracy of the present technique. 
Table 1. Six commonly used boundary conditions.

\begin{tabular}{|c|c|c|c|}
\hline Boundary Conditions & $\alpha_{i}(i=1,4)$ & Values for $\lambda_{m}$ & $\varsigma_{m}$ \\
\hline SS-SS & $\alpha_{1}=\alpha_{2}=\alpha_{3}=0, \quad \alpha_{4}=-1$ & $m \pi$ & 1 \\
\hline $\mathrm{C}-\mathrm{C}$ & $\alpha_{1}=\alpha_{3}=1, \quad \alpha_{2}=\alpha_{4}=-1$ & $\cos \lambda_{m} \cosh \lambda_{m}=1$ & $\frac{\cosh \lambda_{m}-\cos \lambda_{m}}{\sinh \lambda_{m}-\sin \lambda_{m}}$ \\
\hline F-F & $\alpha_{1}=\alpha_{2}=\alpha_{3}=\alpha_{4}=1$ & $\cos \lambda_{m} \cosh \lambda_{m}=1$ & $\frac{\cosh \lambda_{m}-\cos \lambda_{m}}{\sinh \lambda_{m}-\sin \lambda_{m}}$ \\
\hline C-SS & $\alpha_{1}=\alpha_{3}=1, \quad \alpha_{2}=\alpha_{4}=-1$ & $\tan \lambda_{m}=\tanh \lambda_{m}$ & $\frac{\cosh \lambda_{m}-\cos \lambda_{m}}{\sinh \lambda_{m}-\sinh \lambda_{m}}$ \\
\hline C-F & $\alpha_{1}=\alpha_{3}=1, \quad \alpha_{2}=\alpha_{4}=-1$ & $\cos \lambda_{m} \cosh \lambda_{m}=-1$ & $\frac{\sinh \lambda_{m}-\sin \lambda_{m}}{\cosh \lambda_{m}+\cos \lambda_{m}}$ \\
\hline F-SS & $\alpha_{1}=\alpha_{2}=\alpha_{3}=\alpha_{4}=1$ & $\tan \lambda_{m}=\tanh \lambda_{m}$ & $\frac{\cosh \lambda_{m}-\cos \lambda_{m}}{\sinh \lambda_{m}-\sin \lambda_{m}}$ \\
\hline
\end{tabular}

- Simply supported-simply supported (SS-SS)

- Clamped-clamped (C-C)

- Clamped-free (C-F)

\subsection{Isotropic Cylindrical Shells}

In Table 2, the frequency parameters $\Omega=\omega R \sqrt{\left(1-v^{2}\right) \rho / E}$ for an isotropic cylindrical shell is compared with those ones evaluated by Swaddiwudhipong [30] for simply supported boundary conditions. The shell parameters are listed in this table. The comparison is analyzed for the cases: $L / R=20$ and $L / R=0.25$ with circumferential mode $n=1$ to 5 . The absolute differences between the two sets of frequencies are very minute.

In Table 3, frequency parameter $(\Omega)$ for a cylindrical shell with clamped-clamped edge conditions are compared with those evaluated by Joseph and Haim [30] Shell properties are described in the table. It is noticed that for $n \leq 7$, the present frequencies are larger where as for $n \geq 8$, the two frequencies are approximately equal. The fundamental frequency is associated with the circumferential mode number $n=4$.

In Table 4, natural frequencies (Hz) for a clamped-free cylindrical shells are compared with those calculated experimentally by Sewall and Nauman [10] for the axial wave number $m=1,2,3$. Experimental values of the shell frequency are lower than the present theoretical ones. This difference may be due to the some imperfection in the experimental devices. The lowest frequency is associated with the circumferential wave numbers $n=5,8,10$ for $m=1,2,3$ respectively.

\subsection{FGM Cylindrical Shells}

Table 5 represents a comparison of natural frequencies (Hz) for type-I FGM cylindrical shell configured according to those ones evaluated by Loy et al. [18] for simply supported boundary condition and power law exponents $p=0.5,1,5$. The minimum frequency occurs at the circumferential wave number $n=3$ which is about $0.009 \%, 0.001 \%$ and $0.007 \%$ less than those given in [18] whereas in Type II shell, lowest frequency corresponds to circumferential wave number $n=3$ which is about $0.01 \%, 0.009 \%$ and $0.021 \%$, less than those evaluated in [18]. It is concluded from the above comparisons of shell frequencies that the present method is valid and efficient and gives fast and accurate results.

\subsection{Three-Layered FGM Cylindrical Shells with Middle Layer of Isotropic Material}

A three-layered FGM cylindrical shell whose cross section is shown in Figure 1(b), is fabricated in such a way that the inner and outer layers of the shells is fabricated with FGM layers while an isotropic material is inserted at the middle layer of the shell. The thickness of each layer is assumed to be equal. The shell material parameters include the Young's modulus, Poisson's ratio and the mass-density. The Young's modulus is the most in- 
Table 2. Comparison of frequency parameter $\Omega=\omega R \sqrt{\left(1-v^{2}\right) \rho / E}$ for cylindrical shell with simply supported conditions: $h / R=0.002, \quad m=1, E=30 \times 10^{6} \mathrm{lbf} \mathrm{in}^{-2}, \quad v=0.3, \rho=7.35 \times 10^{-4} \mathrm{lbf} \mathrm{s}^{2} \mathrm{in}^{-4}$.

\begin{tabular}{cccc}
\hline$L / R$ & $n$ & Swaddiwudhipong [30] & Present \\
\hline & 1 & 0.016101 & 0.016101 \\
20 & 2 & 0.005453 & 0.005450 \\
& 3 & 0.005042 & 0.005034 \\
& 4 & 0.008534 & 0.008525 \\
& 5 & ----- & 0.013623 \\
& 1 & 0.951993 & 0.951976 \\
& 2 & 0.934461 & 0.934342 \\
& 3 & 0.906732 & 0.906435 \\
& 4 & 0.87076 & 0.870196 \\
& 5 & ----- & 0.827882 \\
\hline
\end{tabular}

Table 3. Comparison of frequency parameter $\Omega=\omega R \sqrt{\left(1-v^{2}\right) \rho / E}$ for cylindrical shell with clamped-clamped conditions: $L=304.8 \mathrm{~mm}, h=0.254 \mathrm{~mm}, R=76.2 \mathrm{~mm}, \quad m=1, \quad E=2.0668 \times 10^{11} \mathrm{~N} / \mathrm{m}^{2}, \quad v=0.3, \quad \rho=7.85 \times 10^{3} \mathrm{Kg} / \mathrm{m}^{3}$.

\begin{tabular}{ccc}
\hline $\boldsymbol{n}$ & Joseph and Haim[30] & Present \\
\hline 3 & 0.1030 & 0.1097 \\
4 & 0.0681 & 0.0715 \\
5 & 0.0515 & 0.0532 \\
6 & 0.0475 & 0.0482 \\
7 & 0.0528 & 0.0529 \\
8 & 0.0639 & 0.0638 \\
9 & 0.0788 & 0.0785 \\
10 & 0.0964 & 0.0960 \\
\hline
\end{tabular}

Table 4. Comparison of natural frequencies $(\mathrm{Hz})$ for cylindrical shell with clamped-free conditions: $R=0.2423 \mathrm{~m}$, $h=0.000648 \mathrm{~m}, L=0.6255 \mathrm{~m}, E=68.95 \times 10^{9} \mathrm{~N} / \mathrm{m}^{2}, v=0.315, \quad \rho=2.7145 \times 10^{3} \mathrm{Kg} / \mathrm{m}^{3}$.

\begin{tabular}{|c|c|c|c|c|c|c|}
\hline \multirow{2}{*}{$n$} & \multicolumn{3}{|c|}{ Sewall and Nauman [10] } & \multicolumn{3}{|c|}{ Present } \\
\hline & $m=1$ & $m=2$ & $m=3$ & $m=1$ & $m=2$ & $m=3$ \\
\hline 2 & --- & --- & --- & 354 & 1500.6 & 2346 \\
\hline 3 & $\begin{array}{l}155.0 \\
157.0\end{array}$ & --- & --- & 182 & 912 & 1715 \\
\hline 4 & 107.0 & --- & --- & 114 & 588 & 1248 \\
\hline 5 & $\begin{array}{l}89.0 \\
91.0\end{array}$ & 341.0 & --- & 95 & 407 & 925 \\
\hline 6 & 102.0 & 276.0 & --- & 106 & 306 & 707 \\
\hline 7 & 130.0 & 240.0 & --- & 134 & 256 & 561 \\
\hline 8 & 166.0 & $\begin{array}{l}227.0 \\
231.0\end{array}$ & --- & 172 & 243 & 469 \\
\hline 9 & 208.0 & 246.0 & 400.0 & 217 & 259 & 418 \\
\hline 10 & 260.0 & 281.0 & --- & 267 & 294 & 403 \\
\hline 11 & 317.0 & 337.0 & $\begin{array}{l}409.0 \\
412.0\end{array}$ & 324 & 341 & 415 \\
\hline 12 & 374.0 & $\begin{array}{l}393.0 \\
396.0\end{array}$ & --- & 385 & 398 & 449 \\
\hline
\end{tabular}


Table 5. Comparison of natural frequencies (Hz) for a simply supported-simply supported type-I, II FGM cylindrical shell: $L / R=20, h / R=0.002, \quad m=1$.

\begin{tabular}{|c|c|c|c|c|c|c|}
\hline \multirow{4}{*}{$n$} & \multicolumn{3}{|c|}{ Loy et al. [18] } & \multicolumn{3}{|c|}{ Present } \\
\hline & \multicolumn{3}{|c|}{$p$} & \multicolumn{3}{|c|}{$p$} \\
\hline & 0.5 & 1.0 & 5.0 & 0.5 & 1.0 & 5.0 \\
\hline & \multicolumn{6}{|c|}{ Type I Shell } \\
\hline 1 & 13.321 & 13.211 & 12.998 & 13.331 & 13.210 & 12.988 \\
\hline 2 & 4.5168 & 4.480 & 4.4068 & 4.5175 & 4.4790 & 4.4045 \\
\hline 3 & 4.1911 & 4.1569 & 4.0891 & 4.1909 & 4.1560 & 4.0883 \\
\hline 4 & 7.0972 & 7.0384 & 6.9251 & 7.0965 & 7.0371 & 6.9244 \\
\hline \multirow[t]{2}{*}{5} & 11.336 & 11.241 & 11.061 & 11.3350 & 11.2404 & 11.0603 \\
\hline & \multicolumn{6}{|c|}{ Type II Shell } \\
\hline 1 & 13.154 & 13.3210 & 13.526 & 13.1545 & 13.3210 & 13.5052 \\
\hline 2 & 4.4550 & 4.5114 & 4.5836 & 4.4550 & 4.5115 & 4.5759 \\
\hline 3 & 4.1309 & 4.1827 & 4.2536 & 4.1308 & 4.1829 & 4.2450 \\
\hline 4 & 7.0076 & 7.0903 & 7.2085 & 7.0034 & 7.0909 & 7.1945 \\
\hline 5 & 11.189 & 11.3293 & 11.516 & 11.1896 & 11.3305 & 11.4944 \\
\hline
\end{tabular}

fluencing the shell vibrations characteristics. In this study the Poisson's ratio is assumed to be constant for functionally graded materials whereas the Young's modulus is a function of the intrinsic thickness variable $(z)$ as well as the Young's moduli of the constituent materials forming functionally graded layers. The thickness of each layer is supposed to be of $h / 3$. This variation of material thickness distribution modifies the stiffness moduli such as:

$$
\begin{aligned}
& A_{i j}=A_{i j}^{\text {in(FGM })}+A_{i j}^{m \text { (isotropic) }}+A_{i j}^{\text {out(FGM })}, \\
& B_{i j}=B_{i j}^{\text {in(FGM })}+B_{i j}^{m \text { (isotropic) }}+B_{i j}^{\text {out(FGM })}, \\
& D_{i j}=D_{i j}^{\text {in(FGM })}+D_{i j}^{m \text { (isotropic) }}+D_{i j}^{\text {out(FGM })} .
\end{aligned}
$$

where $i, j=1,2,6$ and in(FGM), out(FGM) are associated with inner and outer functionally graded layers respectively and $m$ (isotropic) is related with the middle isotropic layer of a cylindrical shell. Their values are given in Appendix I. If we take $M_{1}, M_{2}$ constituent materials at the inner FGM layer and $M_{3}, M_{4}$ at the outer FGM layer, the resultant material properties Young's modulii, Poisson ratios and mass density of inner and outer FGM layers are given as:

$$
\left.\begin{array}{l}
E^{\text {in }(\mathrm{FGM})}=E_{2}-E_{1}\left(3 \frac{z}{h}+\frac{3}{2}\right)^{p}+E_{1} \\
v^{\text {in }(\mathrm{FGM})}=v_{2}-v_{1}\left(3 \frac{z}{h}+\frac{3}{2}\right)^{p}+v_{1} \\
\rho^{\text {in }(\mathrm{FGM})}=\rho_{2}-\rho_{1}\left(3 \frac{z}{h}+\frac{3}{2}\right)^{p}+\rho_{1} \\
E^{\text {out }(\mathrm{FGM})}=E_{4}-E_{3}\left(3 \frac{z}{h}-\frac{1}{2}\right)^{p}+E_{3} \\
v^{\text {out }(\mathrm{FGM})}=v_{4}-v_{3}\left(3 \frac{z}{h}-\frac{1}{2}\right)^{p}+v_{3} \\
\rho^{\text {out }(\mathrm{FGM})}=\rho_{4}-\rho_{3}\left(3 \frac{z}{h}-\frac{1}{2}\right)^{p}+\rho_{3}
\end{array}\right]
$$


By keeping isotropic material at the middle layer and by the variation of the constituents in the FGM layer as shown in Figure 1(b), four types of cylindrical shells can be formed as shown in Table 6. Material properties of the isotropic material as well as FGM constituents are given in the reference [18] [19].

\subsection{Variation of Volume Fractions of FGM Constituents at the Inner and Outer FGM Layers}

Material properties for inner and outer FGM layers of the cylindrical shell vary from $-h / 2$ to $-h / 6$ and from $+h / 6$ to $+h / 2$ respectively. From these relations, one can conclude that at $z=-h / 2$, the effective material properties become $E=E_{1}, v=v_{1}, \rho=\rho_{1}$ whereas for $z=-h / 6$, material properties become $E=E_{2}$, $v=v_{2} \quad \rho=\rho_{2}$ at the inner FGM layer and at $z=+h / 6$, the material properties turn into $E=E_{3}, v=v_{3}$, $\rho=\rho_{3}$ while at $z=+h / 2$, the material properties turn into $E=E_{4}, v=v_{4}, \rho=\rho_{4}$ for functionally graded outer layer of the cylindrical shell.

These results conclude that the material properties vary smoothly and continuously of constituent materials $M_{1}$ and $M_{3}$ from the inner surface to $M_{2}$ and $M_{4}$ to the outer surface of both the FGM layers respectively. Similar response of the material properties is seen in the inverse direction. Variation of volume fractions $V_{1}$, $V_{3}$ and $V_{2}, V_{4}$ of constituent materials $M_{1}, M_{3}$ and $M_{2}, M_{4}$ placed at the inner and the outer shell surfaces at the inner and outer FGM layers respectively of the shell are sketched in Figure 2(a), Figure 2(b) of the three-layered cylindrical shells. In Figure 2(a), variation of volume fractions $V_{1}$ and $V_{2}$ of the constituent materials $M_{1}$ and $M_{2}$ are sketched for the shell inner FGM layer. In this layer, the volume fraction $V_{1}$ of constituent material $M_{1}$ declines from its highest value 1 to its lowest value 0 whereas the volume fraction $V_{2}$ of material $M_{2}$ rises from 0 to 1 in the thickness interval $-0.5 \leq z / h \leq-0.167$. Similar behaviour of volume fractions $V_{3}$ and $V_{4}$ of the FGM constituents $M_{3}$ and $M_{4}$ is shown in Figure 2(b) for the shell outer FGM layer. Volume fraction $V_{3}$ of material $M_{3}$ reduces from 1 to 0 and volume fraction $V_{3}$ of constituent material $M_{3}$ advances from 0 to its maximum value 1 in the thickness variable interval $+0.167 \leq z / h \leq+0.50$ respectively. The middle layer is made up with some isotropic material, whose thickness span over the interval $-0.167 \leq \mathrm{z} / \mathrm{h} \leq+0.167$.

Now to study the influence of power law exponent $p$ on the volume fraction of the constituents in the FGM layers, $M_{1}$ and $M_{3}$ are pure while $M_{2}$ and $M_{4}$ have zero concentration at the inner surface of the inner

Table 6. Configuration of types of FGM cylindrical shells.

\begin{tabular}{cccc}
\hline Type of Shell & Inner FGM layer & Isotropic Layer & Outer FGM layer \\
\hline Shell I & Nickel-Zarconia & Stainless Steel & Nickel-Zarconia \\
Shell II & Nickel-Zarconia & Stainless Steel & Zarconia-Nickel \\
Shell III & Zarconia-Nickel & Stainless Steel & Nickel-Zarconia \\
Shell IV & Zarconia-Nickel & Stainless Steel & Zarconia-Nickel
\end{tabular}

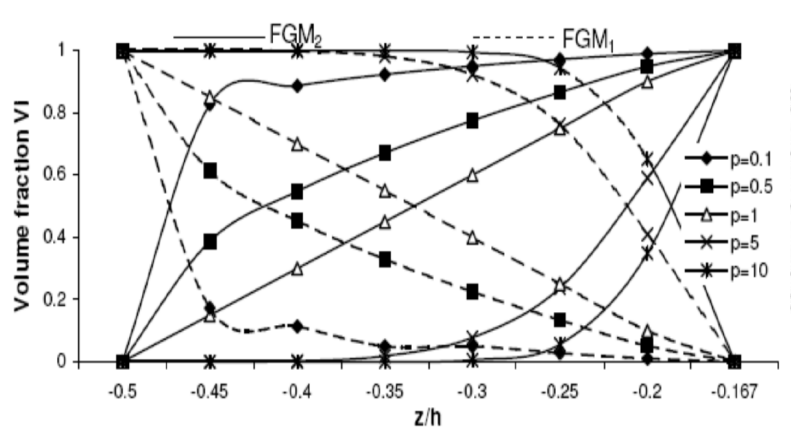

(a)

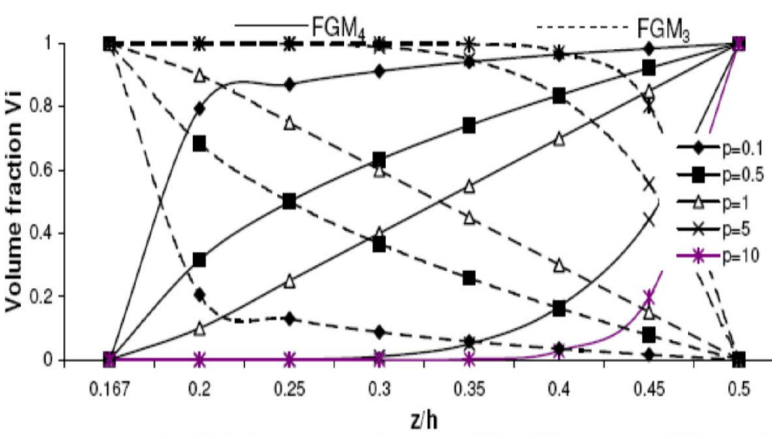

(b)

Figure 2. (a) Variation of volume fractions $\mathrm{FGM}_{1}$ \& $\mathrm{FGM}_{2}$ of Materials $M_{1}$ and $M_{2}$ at the inner FGM layer of the three layered cylindrical shells; (b) Variation of volume fractions $\mathrm{FGM}_{3} \& \mathrm{FGM}_{4}$ of Materials $M_{3}$ and $M_{4}$ at the outer FGM layer of the three layered cylindrical shells. 
and outer FGM layers. Similar but opposite behaviour of the FGM constituents is observed at outer surface of both the layers. For the thickness interval $-0.50 \leq z / h \leq-0.45$ and $p<1, V_{1}$ decreases while $V_{2}$ increase rapidly whereas for $-0.45 \leq z / h \leq-0.167$ and $p<1, V_{1}$ decreases while $V_{2}$ increases slowly and constantly. For the thickness interval $-0.50 \leq z / h \leq-0.25$ and $p>1, V_{1}$ decreases while $V_{2}$ increases gradually but for $-0.25 \leq z / h \leq-0.167$ and $p>1, V_{1}$ decreases and $V_{2}$ increases swiftly. Eventually, $V_{1}$ and $V_{2}$ approach to their minimum and maximum values 0 and 1 respectively. Similar behaviour of volume fractions $V_{3}$ and $V_{4}$ of the constituent materials $M_{3}$ and $M_{4}$ are observed in the outer FGM layer of the three layered cylindrical shell containing middle layer of isotropic material but in this case thickness interval changes from $+0.167 \leq z / h \leq+0.50$.

\subsection{Frequency Analysis of FGM Cylindrical Shell}

In this section variation of natural frequencies $(\mathrm{Hz})$ for four types of shell described in the above table will be discussed. The boundary conditions are taken to be simply supported-simply supported (SS-SS), clamped-clamed (C-C) and clamped-free (C-F).

\subsubsection{Variation of Natural Frequencies $(\mathrm{Hz})$ against Circumferential Wave Number for SS-SS, C-C and C-F Boundary Conditions}

In Table 7 , the variation of natural frequencies $(\mathrm{Hz})$ for three sets of boundary conditions i.e. simply supported-simply supported (SS-SS), clamped-clamped (C-C) and clamped-free(C-F) are studied against the circumferential wave number $n$. In these types of shells the inner and outer layers are composed of the constituents nickel and zirconia while the middle isotropic layer is made of stainless steel. The shell parameters are $L / R=20, h / R=0.002, m=1$ and $p=0.5$. It is seen that the natural frequency first decreases and after at-

Table 7. Variation of natural frequencies $(\mathrm{Hz})$ against circumferential wave number $n$ for shells I, II, III, IV $(m=1, R=1, h=0.002, L=20, p=0.5)$.

\begin{tabular}{|c|c|c|c|}
\hline \multirow{2}{*}{$n$} & SS-SS & $\mathrm{C}-\mathrm{C}$ & $\mathrm{C}-\mathrm{F}$ \\
\hline & \multicolumn{3}{|c|}{ Type I Shell } \\
\hline 1 & 16.1932 & 34.6722 & 6.1921 \\
\hline 2 & 5.4819 & 12.1477 & 2.5145 \\
\hline 3 & 5.0470 & 7.2610 & 4.4855 \\
\hline 4 & 8.5343 & 9.0512 & 8.4259 \\
\hline \multirow[t]{2}{*}{5} & 13.6324 & 13.7689 & 13.6005 \\
\hline & \multicolumn{3}{|c|}{ Type II Shell } \\
\hline 1 & 16.0239 & 34.3095 & 6.1273 \\
\hline 2 & 5.4174 & 12.0174 & 2.4726 \\
\hline 3 & 4.9318 & 7.1420 & 4.3684 \\
\hline 4 & 8.3093 & 8.8288 & 8.2003 \\
\hline 5 & 13.2676 & 13.4052 & 13.2358 \\
\hline \multicolumn{4}{|c|}{ Type III Shell } \\
\hline 1 & 16.0239 & 34.3095 & 6.1273 \\
\hline 2 & 5.4275 & 12.0219 & 2.4946 \\
\hline 3 & 5.0199 & 7.2029 & 4.4673 \\
\hline 4 & 8.5006 & 9.0089 & 8.3940 \\
\hline 5 & 13.5807 & 13.7149 & 13.5494 \\
\hline \multicolumn{4}{|c|}{ Type IV Shell } \\
\hline 1 & 15.8527 & 33.9430 & 6.0619 \\
\hline 2 & 5.3624 & 11.8903 & 2.4524 \\
\hline 3 & 4.9040 & 7.0828 & 4.3497 \\
\hline 4 & 8.2747 & 8.7854 & 8.1676 \\
\hline 5 & 13.2145 & 13.3496 & 13.1832 \\
\hline
\end{tabular}


taining its minimum value it begins to increase with the circumferential wave number $n$. It is also noticed that the natural frequencies for three boundary conditions become closer and closer with the increase of circumferential wave number $n$. The frequencies associated with the clamped-free boundary condition are the lowest among those with the clamped-clamped and simply supported-simply supported boundary conditions. The variation of the frequency is similar to that of an isotropic shell.

\subsubsection{Variation of Natural Frequencies $(\mathrm{Hz})$ against Length-to-Radius Ratio}

In Table 8, the variation of natural frequencies $(\mathrm{Hz})$ against length-to-radius ratio $L / R$ of the four types of three-layered cylindrical shells are studied for three boundary conditions simply supported-simply supported, clamped-clamped and clamped-free. The shell parameters are $h / R=0.002$ with circumferential wave number $n=4$ and the fundamental axial mode $m=1$.

From tables it is observed that the natural frequency $(\mathrm{Hz})$ for all three boundary conditions decreases with the increase of length to radius ratio. It is also seen that the frequencies related to the clamped-clamped boundary conditions are greater than the simply supported-simply supported and clamped-free boundary conditions and the difference between the frequencies for all boundary conditions becomes very negligible at $L / R>20$.

\subsubsection{Variation of Natural Frequencies $(\mathrm{Hz})$ against Thickness-to-Radius Ratio}

In Table 9, variation of natural frequencies of the three-layered cylindrical shell is calculated against the thickness-to-radius ratio under the three selected boundary conditions i.e. simply supported-simply supported, clamped-clamped and clamped-free. In this observation the shell geometrical parameters are $L / R=20$, the fundamental axial mode is $m=1$, and the circumferential wave number $n=4$. From tables it is seen that the

Table 8. Variation of natural frequencies (Hz) against $L / R$ ratios for shell I, II, III, IV $(n=4, m=1, h / R=0.002, p=0.5)$.

\begin{tabular}{|c|c|c|c|}
\hline \multirow{2}{*}{$L / R$} & SS-SS & $\mathrm{C}-\mathrm{C}$ & $\mathrm{C}-\mathrm{F}$ \\
\hline & \multicolumn{3}{|c|}{ Type I Shell } \\
\hline 0.5 & 676.7750 & 720.7210 & 442.8185 \\
\hline 1.0 & 356.1130 & 438.4916 & 171.9393 \\
\hline 5.0 & 23.9693 & 48.6353 & 12.0134 \\
\hline 10 & 10.1874 & 15.5551 & 8.6910 \\
\hline \multirow[t]{2}{*}{50} & 8.4088 & 8.4222 & 8.4054 \\
\hline & \multicolumn{3}{|c|}{ Type II Shell } \\
\hline 0.5 & 669.6757 & 713.1185 & 438.1774 \\
\hline 1.0 & 352.3808 & 433.8956 & 170.1311 \\
\hline 5.0 & 23.6691 & 48.1026 & 11.7902 \\
\hline 10 & 9.9667 & 15.3182 & 8.4666 \\
\hline \multirow[t]{2}{*}{50} & 8.1833 & 8.1968 & 8.1799 \\
\hline & \multicolumn{3}{|c|}{ Type III Shell } \\
\hline 0.5 & 669.7055 & 713.2100 & 438.1912 \\
\hline 1.0 & 352.3917 & 433.9097 & 170.1452 \\
\hline 5.0 & 23.7391 & 48.1366 & 11.9279 \\
\hline 10 & 10.1277 & 15.4230 & 8.6548 \\
\hline \multirow[t]{2}{*}{50} & 8.3771 & 8.3903 & 8.3738 \\
\hline & \multicolumn{3}{|c|}{ Type IV Shell } \\
\hline 0.5 & 662.5305 & 705.5265 & 433.5006 \\
\hline 1.0 & 348.6196 & 429.2646 & 168.3177 \\
\hline 5.0 & 23.4359 & 47.5983 & 11.7031 \\
\hline 10 & 9.9057 & 15.1841 & 8.4294 \\
\hline 50 & 8.1507 & 8.1640 & 8.1474 \\
\hline
\end{tabular}


Table 9. Variation of natural frequencies (Hz) against $h / R$ ratios for shell I. $(n=4, m=1, R=1, L=20, p=0.5)$.

\begin{tabular}{cccc}
\hline$h / R$ & SS-SS & C-C & C-F \\
\hline 0.001 & 4.4440 & Type I Shell & 4.2386 \\
0.005 & 21.0814 & 5.3762 & 21.0286 \\
0.01 & 42.0892 & 21.2855 & 42.0464 \\
0.03 & 126.1878 & 42.1729 & 126.1154 \\
0.05 & 210.2552 & 126.1485 & 210.1423 \\
& & 210.1509 & 4.1260 \\
0.001 & 4.3324 & Type II Shell & 20.4646 \\
0.005 & 20.5174 & 5.2663 & 40.9183 \\
0.01 & 40.9607 & 20.7230 & 122.7320 \\
0.03 & 122.8027 & 41.0458 & 204.5063 \\
0.05 & 204.6163 & 122.7657 & 4.2222 \\
0.001 & & 204.5155 & 20.9494 \\
0.005 & 4.4242 & Type III Shell & 41.8882 \\
0.01 & 21.0015 & 5.3420 & 125.6409 \\
0.03 & 41.9306 & 21.2019 & 209.3512 \\
0.05 & 125.7130 & 42.0126 & 203.6933 \\
\hline 0.001 & 209.4636 & 125.6734 & 4.1091 \\
0.005 & & 209.3594 & 20.3832 \\
0.01 & 4.3121 & Type IV Shell & 40.7558 \\
\hline
\end{tabular}

natural frequencies for all three boundary conditions are very close to each other. For every boundary condition, it is also observed from the tables that the natural frequencies increase as the thickness-to-radius ratio increase.

\section{References}

[1] Yamanouchi, M., Koizumi, M., Hirai, T. and Shiota, I. (1990) Proceedings of the 1st International Symposium on Functionally Gradient Materials, 8-9 October 1990, Sendai.

[2] Rabin, B.H. and Heaps, R.J. (1993) Powder Processing of Ni- $\mathrm{Al}_{2} \mathrm{O}_{3}$ FGM. Ceramic Transactions. Functionally Gradient Materials, 34, 173-180.

[3] Koizumi, M. (1993) The Concept of FGM, Ceramic Transactions. Functionally Gradient Materials, 34, 3-10.

[4] Miyamoto, Y., Kasser, W.A., Rabin, B.H., Kawasaki, A. and Ford, R.G. (1999) Functionally Graded Materials: Design Processing and Applications. Kulwer Academic Publishers, London. http://dx.doi.org/10.1007/978-1-4615-5301-4

[5] Rayleigh, J.W.S. (1882) Theory of Sound. Macmillan, London.

[6] Love, A.E.H. (1888) On the Small Free Vibrations and Deformations of Thin Elastic Shells. Philosophical Transactions of the Royal Society of London, 179A, 125-137.

[7] Arnold, R.N. and Warburton, G.B. (1948) Flexural Vibrations of the Walls of Thin Cylindrical Shells Having Freely Supported Ends. Proceedings of the Royal Society of London, 197, 238-256. http://dx.doi.org/10.1098/rspa.1949.0061

[8] Arnold, R.N. and Warburton, G.B. (1953) The Flexural Vibration of Thin Cylinders. Proceedings of the Institution of Mechanical Engineers, 167, 62-80.

[9] Forsberg, K. (1964) Influence of Boundary Conditions on the Modal Characteristics of Thin Cylindrical Shells. AIAA Journal, 2, 2150-2157. http://dx.doi.org/10.2514/3.55115

[10] Sewall, J.L. and Naumann, E.C. (1968) An Experimental and Analytical Vibration Study of Thin Cylindrical Shells with and without Longitudinal Stiffeners. Langley Research Center, Langley Station, Hampton, Va. NASA TN-4705. 
[11] Warburton, G.B. (1965) Vibration of Thin Cylindrical Shells. Journal of Mechanical Engineering Science, 7, 399-407. http://dx.doi.org/10.1243/JMES_JOUR_1965_007_062_02

[12] Warburton, G.B. and Higgs, J. (1970) Natural Frequencies of Thin Cantilever Cylindrical Shells. Journal of Sound and Vibration, 11, 335-338. http://dx.doi.org/10.1016/S0022-460X(70)80037-2

[13] Goldman, R.L. (1974) Mode Shape and Frequencies Clamped-Clamped Cylindrical Shells. AIAA Journal, 12, 17551756. http://dx.doi.org/10.2514/3.49599

[14] Sharma, C.B. (1974) Calculation of Natural Frequencies of Fixed-Free Circular Cylindrical Shells. Journal of Sound and Vibration, 35, 55-76. http://dx.doi.org/10.1016/0022-460X(74)90038-8

[15] Sharma, C.B. (1979) Vibration Characteristics of Thin Circular Cylinders. Journal of Sound and Vibration, 63, 581592. http://dx.doi.org/10.1016/0022-460X(79)90831-9

[16] Loy, C.T. and Lam, K.Y. (1997) Vibration of Cylindrical Shells with Ring Support. International Journal of Mechanical Sciences, 39, 455-471. http://dx.doi.org/10.1016/S0020-7403(96)00035-5

[17] Naeem, M.N. and Sharma, C.B. (2000) Prediction of Natural Frequencies for Thin Circular Cylindrical Shells. Proceedings of the Institution of Mechanical Engineers, 214, 1313-1328.

[18] Loy, C.T., Lam, K.Y. and Reddy, J.N. (1999) Vibration of Functionally Graded Cylindrical Shells. International Journal of Mechanical Sciences, 41, 309-324. http://dx.doi.org/10.1016/S0020-7403(98)00054-X

[19] Pradhan, S.C., Loy, C.T., Lam, K.Y. and Reddy, J.N. (2000) Vibration Characteristics of Functionally Graded Cylindrical Shells under Various Boundary Conditions. Applied Acoustics, 61, 111-129. http://dx.doi.org/10.1016/S0003-682X(99)00063-8

[20] Arshad, S.H., Naeem, M.N. and Sultana, N. (2007) Frequency Analysis of Functionally Graded Material Cylindrical Shells with Various Volume Fraction Laws. Proceedings of the Institution of Mechanical Engineers, Part C: Journal of Mechanical Engineering Science, 221, 1483-1495. http://dx.doi.org/10.1243/09544062JMES738

[21] Arshad, S.H., Naeem, M.N., Sultana, N., Iqbal, Z. and Shah, A.G. (2011) Effects of Exponential Volume Fraction Law on the Natural Frequencies of Functionally Graded Materials Cylindrical Shells under Various Boundary Conditions. Archive of Applied Mechanics, 81, 999-1016. http://dx.doi.org/10.1007/s00419-010-0460-5

[22] Najafizadeh, M.M. and Isvandzibaei, M.R. (2007) Vibration of Functionally Graded Cylindrical Shells Based on Higher Order with Shear Deformation Plate Theory with Ring Support. Acta Mechanica, 191, 75-91. http://dx.doi.org/10.1007/s00707-006-0438-0

[23] Sofiyev, A.H., Deniz, A., Akeay, I.H. and Yusufoglu, E. (2006) The Vibration and Stability of a Three-Layered Conical Shell Containing FGM Layer Subject to Axial Compression Load. Acta Mechanica, 183, 129-144. http://dx.doi.org/10.1007/s00707-006-0328-5

[24] Sofiyev, A.H. (2007) Vibration and Stability of Composite Cylindrical Shells Containing a FG Layer Subject to Various Loads. Structural Engineering and Mechanics: An International Journal, 27, 365-391.

[25] Li, S.R. and Batra, R.C. (2005) Buckling of a Laminated Cylindrical Shell with Functionally Graded Middle Layer under Axial Compressive Load. Proc. of the Int. Conf. on Mech. Engng and Mech, 1-2, 796-800.

[26] Li, S.R. and Batra, R.C. (2006) Buckling of Axially Compressed Thin Cylindrical Shells with Functionally Graded Middle Layer. Thin-Walled Structures, 44, 1039-1047. http://dx.doi.org/10.1016/j.tws.2006.10.006

[27] Toulokian, Y.S. and Powell, R.W. (1973) Thermal Conductivities of the Elements. Science, 181, 999-1008. http://dx.doi.org/10.1126/science.181.4104.999

[28] Gasser, L.F.F. (1987) Free Vibrations on Thin Cylindrical Shells Containing Liquid. M.S. Thesis, Federal Univ. of Rio de Janerio, peccoppe-ufrj, Rio de Janerio. (In Portuguese)

[29] Swaddiwudhipong, S., Tian, J. and Wang, C.M. (1995) Vibration of Cylindrical Shells with Intermediate Supports. Journal of Sound and Vibration, 187, 69-93. http://dx.doi.org/10.1006/jsvi.1995.0503

[30] Callanhan, J. and Baruh, H. (1999) A Closed-Form Solution Procedure for Circular Cylindrical Shell Vibrations. International Journal of Solids and Structures, 36, 2973-3013. http://dx.doi.org/10.1016/S0020-7683(98)00139-5 
M. N. Naeem et al.

Appendix

$$
\begin{gathered}
C_{11}=A_{11} I_{1}+\frac{n^{2}}{R^{2}} A_{66} I_{2} \\
C_{12}=\frac{n}{R}\left(A_{12}+\frac{1}{R} B_{12}\right) I_{3}-\frac{n}{R}\left(A_{66}+\frac{2}{R} B_{66}\right) I_{2} \\
C_{13}=\frac{1}{R}\left(A_{12}+\frac{n^{2}}{R} B_{12}\right) I_{3}-B_{11} I_{1}-\frac{2 n^{2}}{R^{2}} B_{66} I_{2} \\
C_{22}=\frac{n^{2}}{R^{2}}\left(A_{22}+\frac{2}{R} B_{22}+\frac{1}{R^{2}} D_{22}\right) I_{4}+\left(A_{66}+\frac{4}{R} B_{66}+\frac{4}{R^{2}} D_{66}\right) I_{2} \\
\left.C_{33}=\frac{1}{R^{2}}\left(A_{22}+\frac{1+n^{2}}{R} B_{22}+\frac{n^{2}}{R^{2}} D_{22}\right) I_{4}-\frac{n}{R}\left(B_{12}+\frac{1}{R} D_{12}\right) I_{3}+\frac{2 n}{R}\left(B_{66}+\frac{n^{4}}{R} D_{66}\right) I_{2}\right) I_{4}-\frac{2}{R}\left(B_{12}+\frac{n}{R} D_{12}\right) I_{3}+D_{11} I_{1}+\frac{4 n^{2}}{R^{2}} D_{66} I_{2}
\end{gathered}
$$

where

$$
\begin{gathered}
I_{1}=\frac{m^{4} \pi^{4}}{2 L^{3}}, I_{2}=\frac{m^{2} \pi^{2}}{2 L} \\
I_{3}=-\frac{m^{2} \pi^{2}}{2 L}, I_{4}=\frac{L}{2} \\
A_{11}=A_{22}=\frac{h}{3\left(1-v^{2}\right)}\left(\frac{E_{1}-E_{2}}{p+1}+E_{2}\right)+\frac{h E}{3\left(1-v^{2}\right)}+\frac{h}{3\left(1-v^{2}\right)}\left(\frac{E_{3}-E_{4}}{p+1}+E_{4}\right) \\
A_{12}=\frac{h v}{3\left(1-v^{2}\right)}\left(\frac{E_{1}-E_{2}}{p+1}+E_{2}\right)+\frac{h v E}{3\left(1-v^{2}\right)}+\frac{h v}{3\left(1-v^{2}\right)}\left(\frac{E_{3}-E_{4}}{p+1}+E_{4}\right) \\
A_{66}=\frac{h}{6(1+v)}\left(\frac{E_{1}-E_{2}}{p+1}+E_{2}\right)+\frac{h E}{6(1+v)}+\frac{h}{6(1+v)}\left(\frac{E_{3}-E_{4}}{p+1}+E_{4}\right) \\
B_{11}=B_{22}=\frac{-h^{2}}{9\left(1-v^{2}\right)}\left(\frac{\left(E_{1}-E_{2}\right)(p+4)}{2\left(p^{2}+3 p+2\right)}+E_{2}\right)+\frac{h^{2}}{9\left(1-v^{2}\right)}\left(\frac{\left(E_{3}-E_{4}\right)(3 p+4)}{2\left(p^{2}+3 p+2\right)}+E_{4}\right) \\
B_{12}=\frac{-h^{2} v}{9\left(1-v^{2}\right)}\left(\frac{\left(E_{1}-E_{2}\right)(p+4)}{2\left(p^{2}+3 p+2\right)}+E_{2}\right)+\frac{h^{2} v}{9\left(1-v^{2}\right)}\left(\frac{\left(E_{3}-E_{4}\right)(3 p+4)}{2\left(p^{2}+3 p+2\right)}+E_{4}\right) \\
B_{66}=\frac{-h^{2}}{18(1+v)}\left(\frac{\left(E_{1}-E_{2}\right)(p+4)}{2\left(p^{2}+3 p+2\right)}+E_{2}\right)+\frac{h^{2}}{18(1+v)}\left(\frac{\left(E_{3}-E_{4}\right)(3 p+4)}{2\left(p^{2}+3 p+2\right)}+E_{4}\right) \\
D_{11}=D_{22}=\frac{h^{3}}{108\left(1-v^{2}\right)}\left(\frac{\left(E_{1}-E_{2}\right)\left(p^{2}+9 p+26\right)}{p^{3}+6 p^{2}+11 p+6}+\frac{13}{3} E_{2}\right)+\frac{h^{3} E}{324\left(1-v^{2}\right)} \\
+\frac{h^{3}}{108\left(1-v^{2}\right)}\left(\frac{\left(E_{3}-E_{4}\right)\left(p^{2}+33 p+26\right)}{p^{3}+6 p^{2}+11 p+6}+\frac{13}{3} E_{4}\right) .
\end{gathered}
$$

330 


$$
\begin{aligned}
& D_{12}= \frac{h^{3} v}{108\left(1-v^{2}\right)}\left(\frac{\left(E_{1}-E_{2}\right)\left(p^{2}+9 p+26\right)}{p^{3}+6 p^{2}+11 p+6}+\frac{13}{3} E_{2}\right)+\frac{h^{3} E v}{324\left(1-v^{2}\right)} \\
&+\frac{h^{3} v}{108\left(1-v^{2}\right)}\left(\frac{\left(E_{3}-E_{4}\right)\left(p^{2}+33 p+26\right)}{p^{3}+6 p^{2}+11 p+6}+\frac{13}{3} E_{4}\right) . \\
& D_{66}= \frac{h^{3}}{216(1+v)}\left(\frac{\left(E_{1}-E_{2}\right)\left(p^{2}+9 p+26\right)}{p^{3}+6 p^{2}+11 p+6}+\frac{13}{3} E_{2}\right)+\frac{h^{3} E}{648(1+v)} \\
& \quad+\frac{h^{3}}{216(1+v)}\left(\frac{\left(E_{3}-E_{4}\right)\left(9 p^{2}+33 p+26\right)}{p^{3}+6 p^{2}+11 p+6}+\frac{13}{3} E_{4}\right) .
\end{aligned}
$$


Scientific Research Publishing (SCIRP) is one of the largest Open Access journal publishers. It is currently publishing more than 200 open access, online, peer-reviewed journals covering a wide range of academic disciplines. SCIRP serves the worldwide academic communities and contributes to the progress and application of science with its publication.

Other selected journals from SCIRP are listed as below. Submit your manuscript to us via either submit@scirp.org or Online Submission Portal.
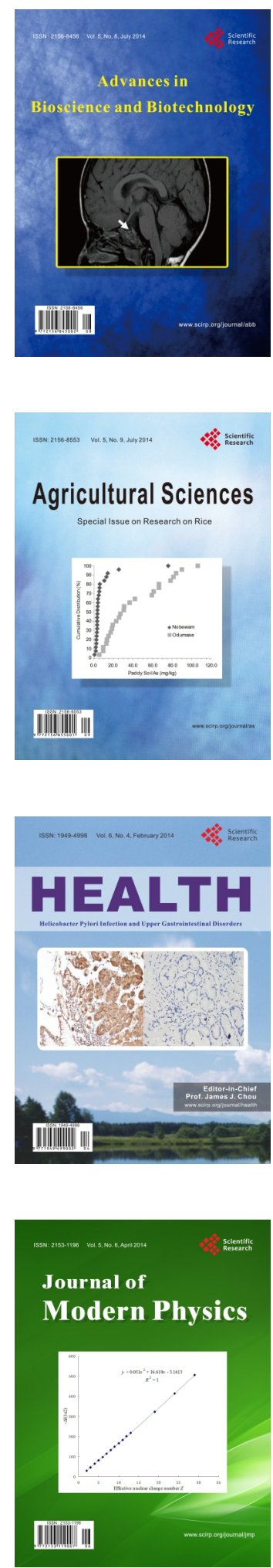
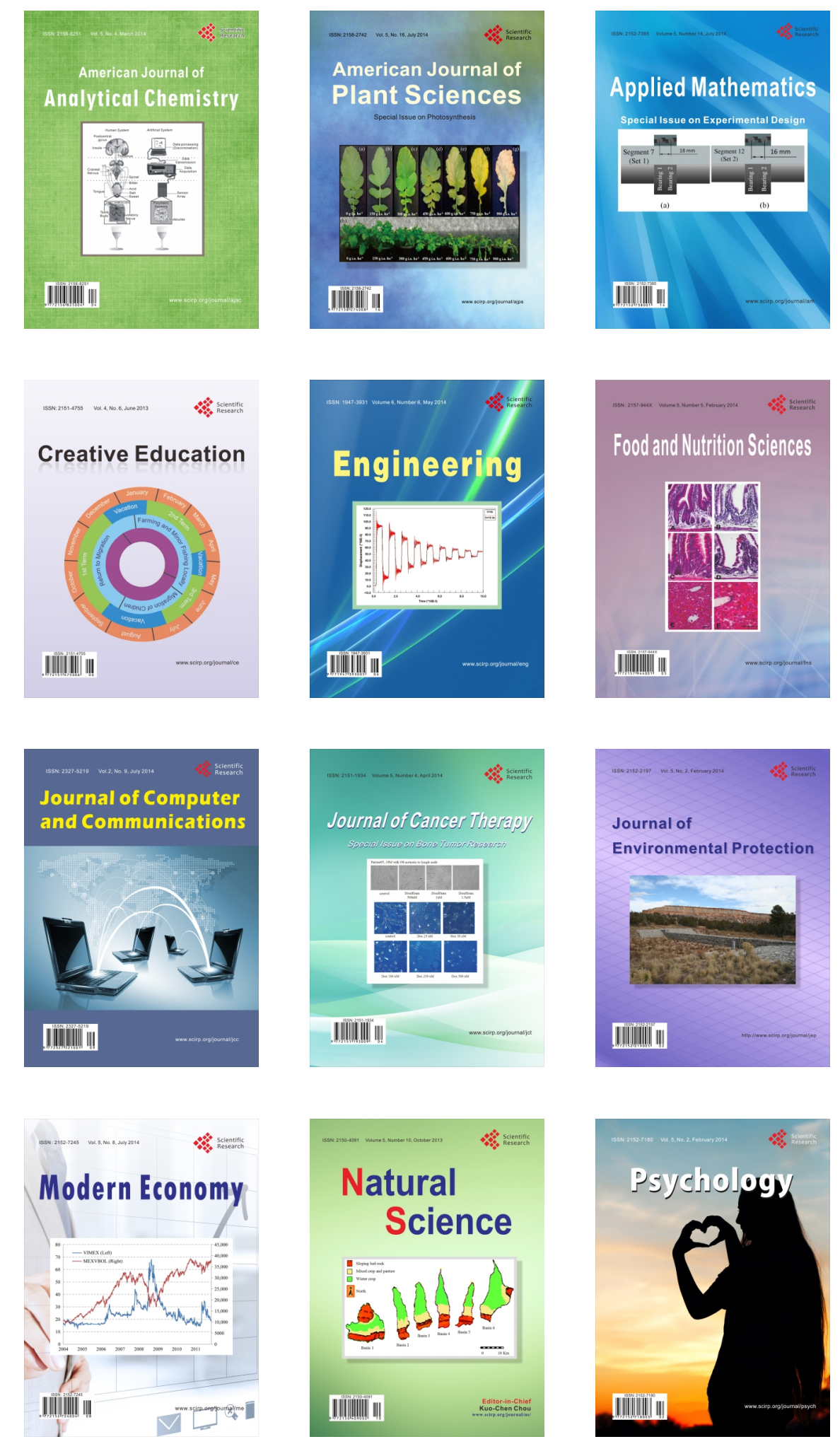